
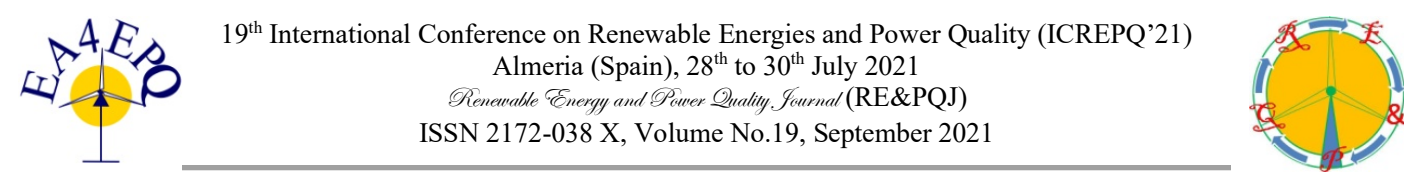

\title{
Development of a power transformer model for high-frequency transient phenomena
}

\author{
L. Braña ${ }^{12}$, A. Costa $^{2}$ and R. Lopes ${ }^{1}$ \\ 1. $\quad$ Efacec Transformers R\&D - Apartado 1018 - 4466-952 S. Mamede de Infesta, Portugal \\ e-mail: luis.brana@efacec.com, ricardol@efacec.com \\ ${ }^{2}$ Department of Electrical Engineering \\ FEUP, University of Porto \\ Rua Dr. Roberto Frias, 4200-465 Porto, Portugal \\ e-mail: acosta@fe.up.pt
}

\begin{abstract}
In recent years, the proliferation of distributed renewable energy sources and the application of new rules for the exploitation of electrical networks imposed by the markets have dictated increasingly demanding operating conditions for electric power transformers, creating new challenges in their exploration and conservation. Transformers that, in addition to the transmission lines, are certainly the most important and critical element of any electrical energy system.

Adequate models are necessary to accurately describe transformer behavior and internal response when submitted to different external requests imposed by the network, particularly during transient phenomena, as well as, to properly assess system vulnerabilities and network optimization. This effort is being carried out today by several research groups in the world, namely from Cigré and IEEE.

In this work, a transformer model to be integrated into a timedomain equivalent circuit is developed and discussed. Results obtained with this model are compared with measurements obtained by the Cigré JWG A2/C4.52 in a power transformer used as a reference for the working group.
\end{abstract}

Key words. Power transformer, white-box model, electromagnetic transients, voltage transfer measurement, simulation.

\section{Introduction}

The transformer isolation design is one of the most important aspects of the design step since it is an essential point which determines the life expectancy of the transformer. The isolation structure is designed according to the voltage distribution along the windings. When operating, the isolation structure is continuously exposed to the rated voltage and, occasionally, to overvoltages as a consequence of the interaction between the transformer and the network. Although there is a great knowledge of the transformer behavior at rated voltage, the same is not exactly true for the transient phenomena resulting from an interaction between the grid and the transformer.
Therefore, it is necessary to have new tools to accurately model and reproduce the response of the internal transformer in these more demanding conditions, with different approaches to achieve this.

The proposed model is based on a lumped parameter network which represents in detail the internal geometry of the transformer in the so-called white-box model. This model is broadly used by transformer manufacturers in the design stage.

The model parameters are based on the transformer geometry considering magnetic core, tank and windings, as well as its material properties. These parameters include inductances, capacitances and resistances.

At very high frequencies, transformer windings behave as electric transmission lines with dissipative and coupled parameters, where the theory of the electromagnetic waves in lossy transmission lines can be applied. As shown in [1], each winding is split into a different number of blocks which are represented as Pi equivalent circuits. Parameters of each block are obtained by grouping the parameters of several turns.

\section{White-Box Modelling}

The transformer manufacturers make use of detailed models, the so-called white-box models, for predicting the internal voltage stresses that arise when applying the standard lightning impulse voltage to external terminals. A generic transformer model is extremely complex due to the great design variety of the magnetic core and the windings.

The main concept of white-box modelling is to represent the transformer topology through passive elements in an electrical circuit. 
A typical modern approach considers a lumped parameter model in which some parameters (capacitances and resistances) are calculated by analytical methods and the inductances are calculated by the finite element method [2]. Some parameters have nonlinear behavior and others are frequency dependents. Resistances describe DC and eddy losses induced by skin and proximity effect in the turns. Inductances represent the leakage flux due to the magnetic coupling of the turns among windings - namely self and mutual inductance. Finally, capacitors model the electrical coupling due to the capacitive effect of the insulation.

The model is supposed to simulate with accuracy a transformer within a frequency range if the parameters are well determined [3], which usually occurs in the low and mid-frequency range (from zero to few $\mathrm{kHz}$ ). Besides this range, out of the normal operation limits, transformer parameters are usually unknown, even for the manufacturers.

In our study, a lumped-parameter white-box model of the transformer similar to the one shown in Fig 1 has been created based on detailed design information about the transformer and spatial discretization, giving a description in terms of capacitance, inductance and resistance matrices, and internal connections.

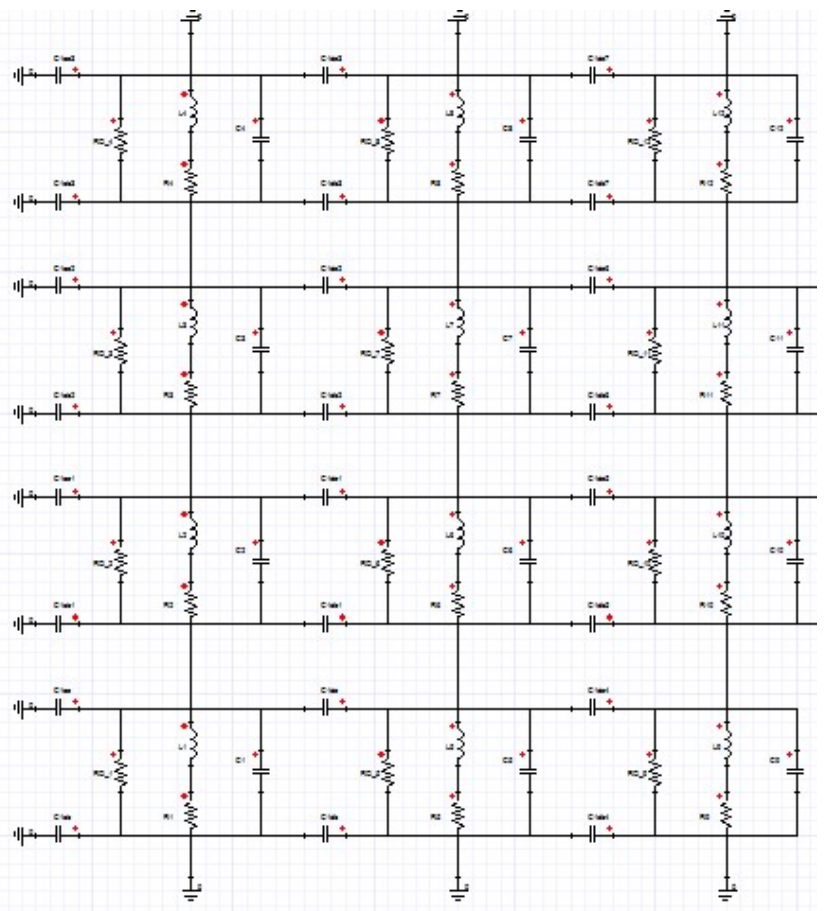

Fig 1. Transformer model.

Every winding is constructed by cells as depicted in Fig. 2. A cell can represent a group of disks or a single one, depending on the desired accuracy. The greater number of cells, the more accurate and slower is the solving time of the model.

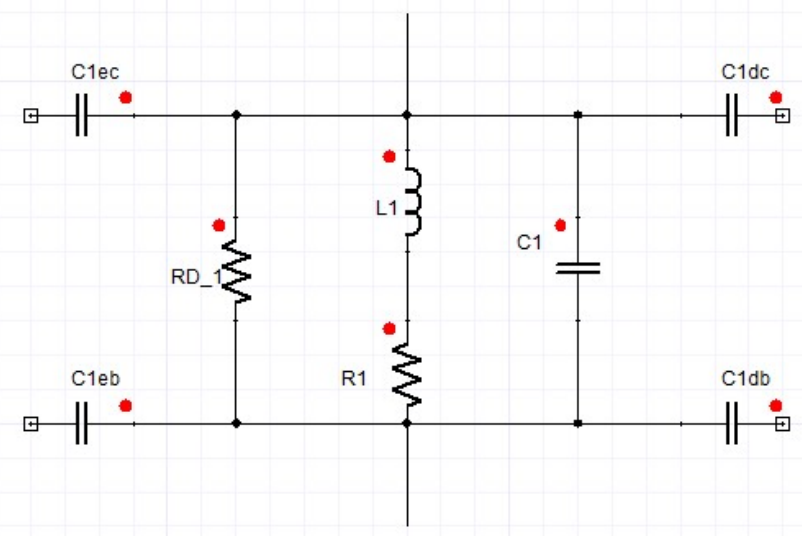

Fig 2. Detailed cell.

In the cell depicted in Fig. 2, $L 1$ represents the inductance of the cells and has been calculated by using the finite element method for a 2D axisymmetric winding. C1 represents the series/axial capacitance along the cell and has been calculated by an analytic formula modified by empiric factors developed by the transformer manufacturer. $C 1 e b / e c / d c / d c$ represents the radial/parallel capacitance between windings and between windings and structural parts, namely magnetic core and tank. These capacitances have been calculated by adapted formulas of the cylindric capacitor and they have been verified in a finite element model. $R 1$ represents the losses associated (eddy and DC) and has been calculated by the analytic method described in [4]. At last, $R D_{-} 1$ is the resistance which governs the damping of the impulse wave and has been calculated through an empiric formula based on experimental data. Parameters are not frequency dependent and were calculated for frequencies above 10 $\mathrm{kHz}$, where it is supposed the magnetic core does not influence the transformer performance.

\section{Experimental Tests}

\section{A. Transformer Data}

The tested transformer is depicted in Fig 2 and Fig. 3. It is a three-leg, 50 MVA, core-type, one-phase power transformer with a rated frequency of $60 \mathrm{~Hz}$. The rated voltages are $220 \mathrm{kV}, 69 \mathrm{kV}$ and $50 \mathrm{kV}$, for the high voltage, low voltage, and tertiary voltage respectively with connection YNynd. Bushing Insulation Level for High Voltage is AC $395 \mathrm{kV}_{\text {rms }} /$ SI $850 \mathrm{kV}_{\text {peak }} /$ LI 1050 $\mathrm{kV}_{\text {peak. }}$.

The transformer is built with 335 coils ( 83 for tertiary winding, 128 for LV winding, 124 for $\mathrm{HV}$ winding and 80 for regulating winding). 


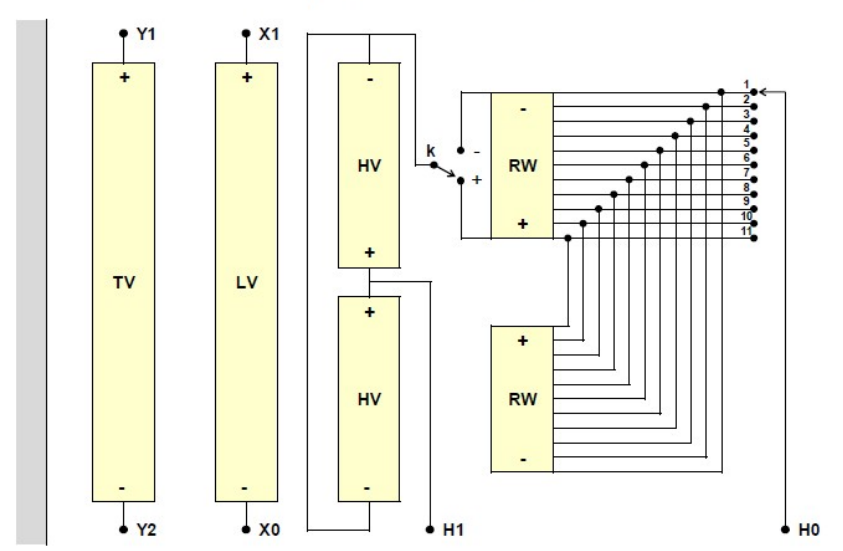

Fig 2. Polarities and connections layout.

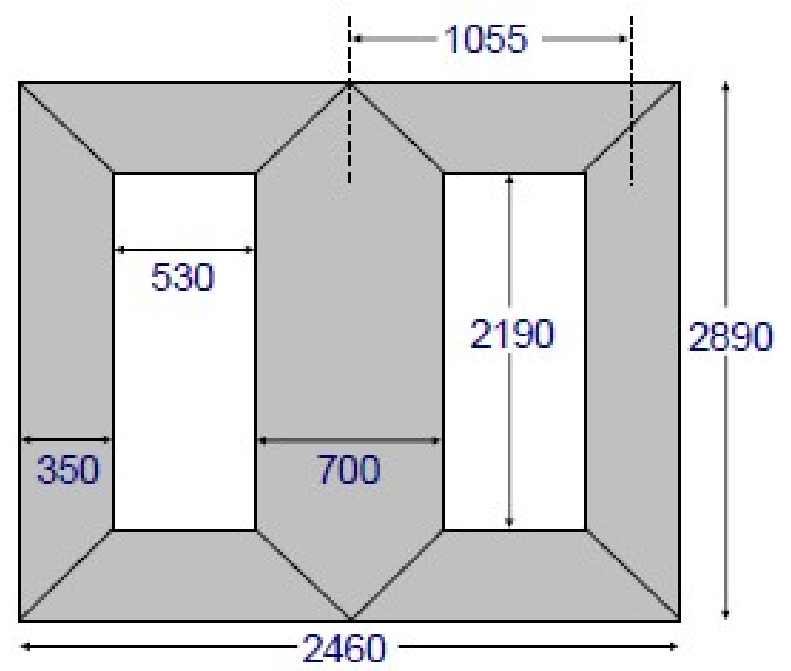

Fig 3. Magnetic core design.

\section{B. Voltage Transfer Measurements}

Assessment of internal stresses due to any non-standard voltage wave shapes and terminal conditions can only be performed by using a high-frequency transformer model available to the transformer manufacturer. The highfrequency component of this model can be validated, under certain conditions, through Small Signal Internal Voltage Transfer Measurement Tests.

These tests included non-standard terminal connections with open terminals, differing from those tests defined by the international standards related to impulse testing of power transformers and they are suitable for assessing the white box model performance at higher frequencies, where transformer parameters present greater uncertainty and, in turn, will allow readjusting the model parameters for simulating any transient phenomena in the high frequency range, f.e. a lightning overvoltage, [5]. The test voltage responses were obtained using voltage transfer frequency sweep measurements that were converted into timedomain waveforms.
The basic approach is to measure the voltage transfer function from one transformer terminal to a second terminal, as a function of discrete frequency. The voltage transfer function $h(\omega)$ is defined as the response voltage $V 2(\omega)$ divided by the excitation voltage $V 1(\omega)$,

$$
h(\omega)=\frac{V_{2}(\omega)}{V_{1}(\omega)}
$$

The voltage transfer measurements were performed using two identical passive voltage probes in combination with a vector network analyzer (VNA). A coaxial cable was connected from the VNA output to the transformer terminal to be excited, with the shield grounded on the tank rim ground reference (in addition to the VNA ground). One voltage probe was connected from the VNA reference to the excited terminal, with the ground clip connected to the ground reference. The other voltage probe was connected from the VNA input to the terminal where the voltage response was to be measured, with the ground clip connected to the ground reference. The VNA then performed a frequency sweep measurement of the input voltage divided by the reference voltage, directly giving the voltage transfer function.

The measurements were performed with the transformer active parts inside the tank, but without oil and bushings. A flat braided wire was clamped to the tank rim and used as the ground reference in the measurements. The winding terminals were brought to the rim and connected to suitable test fixtures which permitted easy grounding, connection of voltage probes, and connection of shielded cables for admittance measurements. In addition, unshielded cables were connected to three points in the regulating winding (extreme ends and close to mid-point) that were brought to the tank rim.

A large set of measurements of node-ground voltages were performed, representing voltage transfer between external terminals. In addition, the voltage transfer from external terminals to three points (R1, R5, R11) inside the tap changer was measured. Fig. 4 shows in the first four rows the basic measurement cases which will be shown in this work. These four cases were performed with four alternative tap settings (Max, Nom+, Nom- and Min), and with the excitation in either $\mathrm{H} 1, \mathrm{H} 0, \mathrm{X} 1$ or $\mathrm{X} 0$, giving a total of $4 \times 4 \times 4=64$ cases.

The specifications shown in Fig. 4 are graphically represented in Fig. 5, where Case 1 has been taken as an example. 


\begin{tabular}{|c|c|c|c|c|c|c|c|c|c|c|c|c|c|c|c|c|c|c|c|}
\hline \multirow{2}{*}{ Case } & \multirow{2}{*}{$\begin{array}{c}\text { Tap } \\
\text { Position }\end{array}$} & \multirow{2}{*}{ Y2 } & \multirow{2}{*}{ Y1 } & \multirow{2}{*}{$\mathrm{X}_{0}$} & \multirow{2}{*}{ X1 } & \multirow{2}{*}{ H1 } & \multirow{2}{*}{ HO } & \multicolumn{12}{|c|}{ Measurements } \\
\hline & & & & & & & & H1 & $\mathrm{HO}$ & $\mathrm{X} 1$ & $\mathrm{X} 0$ & $\mathrm{Y} 1$ & Y2 & R1 & R5 & R11 & R1-R11 & $\mathrm{k}-\mathrm{X} 1$ & $\mathrm{X} 1-\mathrm{Y1}$ \\
\hline 1 & Max & Grounded & Grounded & Grounded & Grounded & Applied & Grounded & Yes & & & & & & & Yes & & Yes & Yes & \\
\hline 17 & $\operatorname{Max}$ & Grounded & Grounded & Grounded & Grounded & Grounded & Applied & & Yes & & & & & & Yes & Yes & Yes & Yes & \\
\hline 33 & $\operatorname{Max}$ & Grounded & Grounded & Grounded & Applied & Grounded & Grounded & & & Yes & & & & & Yes & & Yes & Yes & \\
\hline
\end{tabular}

Fig 4. Terminal conditions and measurements test (4 out of 64 cases).
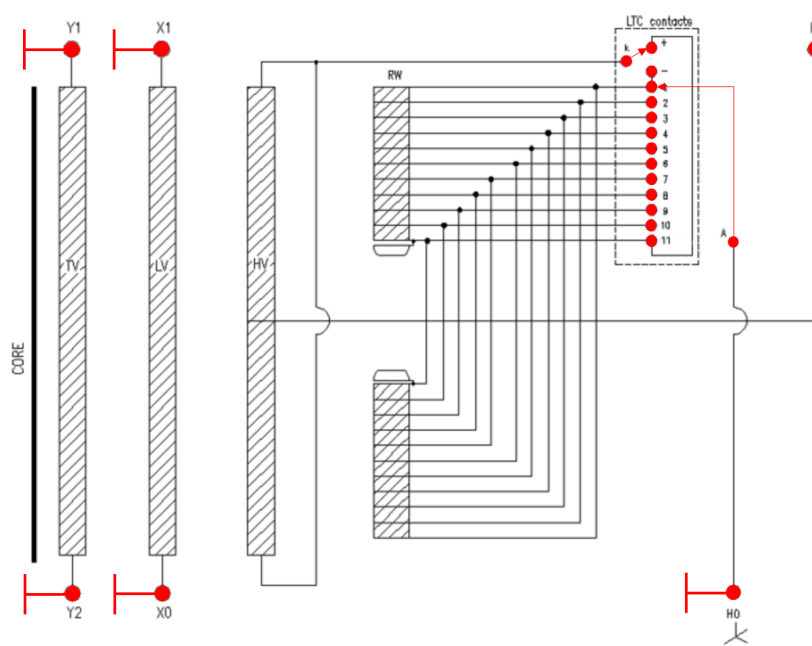

Fig 5. Scheme of connections for case 1.

Due to the large number of voltage transfer measurements, it was considered impractical to do the measurements in the time domain. It was foreseen that the voltage excitation would vary from case to case due to the difference in transformer input impedance at the excited terminals. It was therefore decided to perform the voltage transfer measurements in the frequency domain and afterward calculate time domain voltage waveforms for alternative excitations using convolution. Using the rational approximation (2), which defines an impulse response, the time domain response of any time domain excitation can be calculated using "recursive convolution" via time domain discretization. Details of the rational fitting and time domain convolution are found in [6] and reference therein.

$$
h(\omega) \cong \sum_{i=1}^{N} \frac{\gamma_{1}}{j \omega-\sigma_{\bar{L}}}
$$

Where $h(\omega)$ represents the impulse voltage response, $a_{i}$ and $r_{i}$ are real or conjugate complex which represent the poles and residues respectively.

\section{Results}

Voltage transferred measurements were performed between different terminal points applying voltage with standardized shape $(1.2 / 50 \mu \mathrm{S})$ as depicted in Fig. 6 .

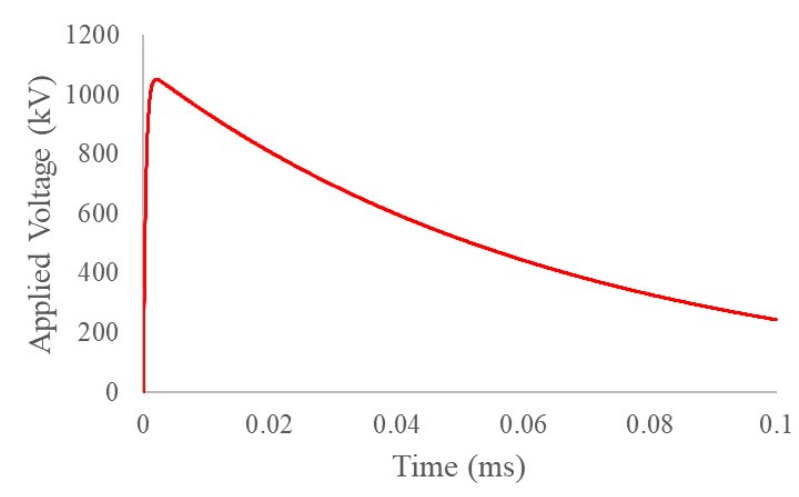

Fig 6. Impulse voltage wave.

Figs. 7, 8, 9 and 10 show the comparison between the calculated values and the measured results.

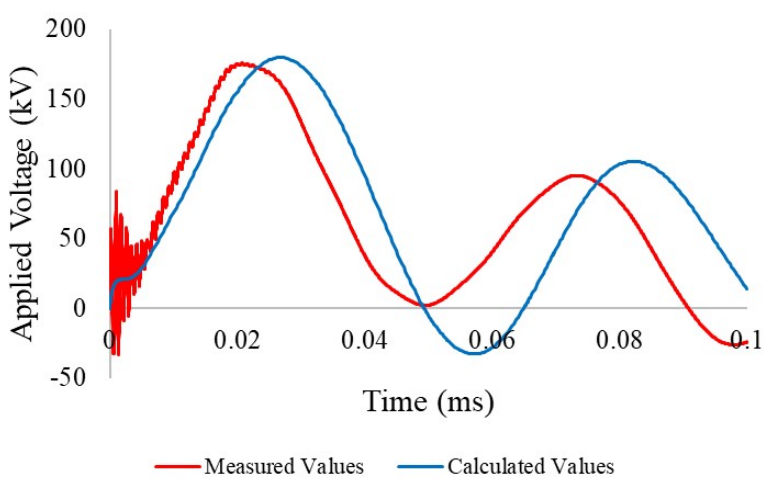

Fig. 7. Comparison for Case 01, R11 lead, (red: measurement, blue: calculation)

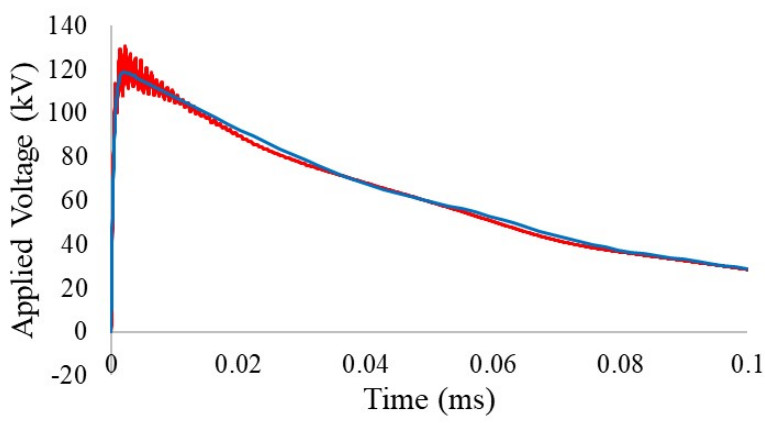

—Measured Values Calculated Values

Fig. 8. Comparison for Case 17, R5 lead, (red: measurement, blue: calculation) 


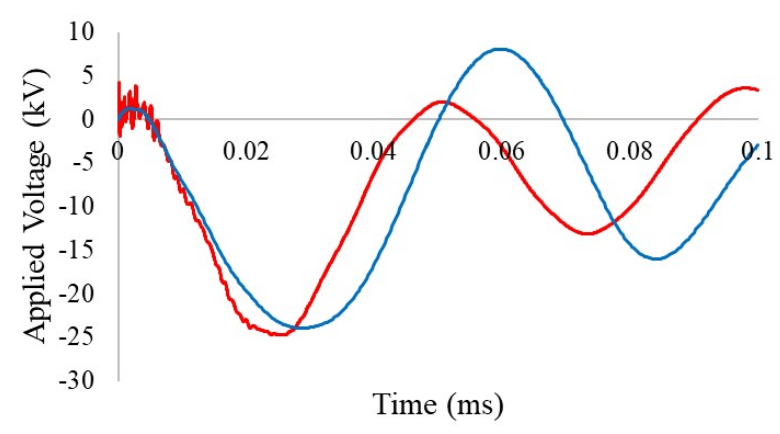

- Measured Values Calculated Values

Fig. 9. Comparison for Case 33, R5 lead, (red: measurement, blue: calculation)

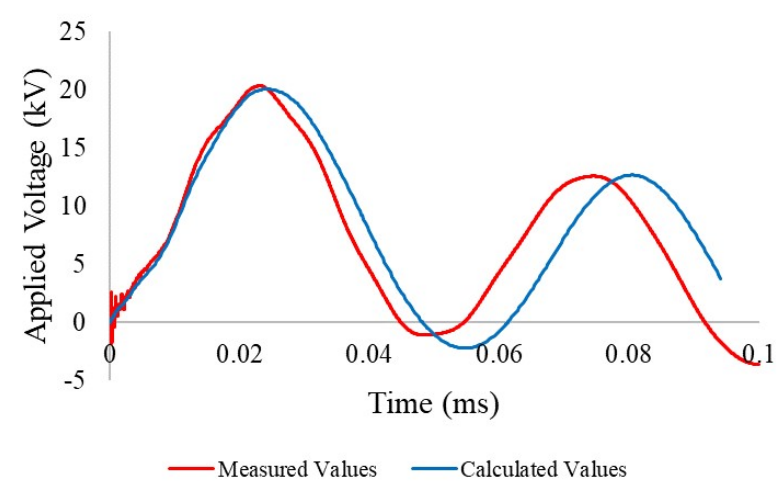

Fig. 10. Comparison for Case 49, R11 lead, (red: measurement, blue: calculation)

In all cases, a good agreement was obtained between the measured transferred wave amplitude and the calculated by the white box model for the first response periods. Although the phase differences, the voltage peaks are predicted with reasonable accuracy. Therefore, from the transformer manufacturer prospect, results are acceptable since they allow us to predict the critical value for the design of the isolation structures. It should be considered that the model developed has been thought for all the voltage range of power transformers so empirical factors have not been calibrated for this single transformer.

Anyway, several actions should be taken in order to increase the accuracy of the model:

- The accuracy of the wave in the decaying region can be improved by recalibrating the value of the resistance $R D_{-} 1$;

- Serie capacitance for the interleaved winding should be reviewed. Since in this winding the consecutive electric turns are separated by another turn, there are some uncertainties in the turn-to-turn capacitance calculation;

- In an ideal scenario, all parameters should be frequency dependent.

\section{Conclusions}

This work has demonstrated that a white-box model approach can accurately simulate the response to highfrequency tests, which validates its use for simulating transformer high-frequency transient response.

A non-standard impulse test procedure has been used for obtaining the voltage response at critical positions in the regulating winding, with alternative terminal conditions. The measured responses were compared to simulations by a white-box model, demonstrating a good accuracy between measurements and simulations. The measurements are in general fast and easy to perform.

The approach is an excellent means of assessing the accuracy of a manufacturer's impulse voltage computational tools, thereby enabling further improvements to the model's parameter determination.

\section{Acknowledgement}

This work has been co-funded under the European Framework P2020 incentive program to the Portuguese Industry. The project acronym is GreenEst (Project POCI01-0247-FEDER-033811).

\section{References}

[1] C. Álvarez Mariño, 'Estudio de la respuesta en muy alta frecuencia en transformadores de potencia', http://purl.org/dc/dcmitype/Text, Universidade de Vigo, 2014.

[2] E. Bjerkan and H. K. Høidalen, 'High frequency FEMbased power transformer modeling: Investigation of internal stresses due to network-initiated overvoltages', Electr. Power Syst. Res., vol. 77, no. 11, pp. 1483-1489, Sep. 2007, doi: 10.1016/j.epsr.2006.08.031.

[3] S. Cho, 'Parameter estimation for transformer modeling', Diss. Masters Theses Masters Rep. - Open, Jan. 2002, doi: 10.37099/mtu.dc.etds/60.

[4] E. Bjerkan, 'High Frequency Model of Power Transformers', Norwegian University of Science and Technology, 2005.

[5] B. Gustavsen, A. Portillo, R. Ronchi, and A. Mjelve, 'High-Frequency Modeling and Simulation of a SinglePhase Three-Winding Transformer Including Taps in Regulating Winding', Nov. 2016.

[6] B. Gustavsen, A. Portillo, R. Ronchi, and A. Mjelve, 'Measurements for validation of manufacturer's white-box transformer models', May 2017. 